\title{
ELECTRICALLY DETECTED ELECTRON PARAMAGNETIC RESONANCE
}

\section{S. GREULICH-WEBER}

University of Paderborn, FB6, Warburger Str. 100, 33098 Paderborn, Germany

Key words: EDEPR, recombination centres

ABSTRACT. Commercial silicon diodes and bulk silicon were investigated with electrically detected electron paramagnetic resonance (EDEPR). We observed deep as well as shallow recombination centres with different defect concentrations. Different ways of EPR detection were studied such as electrical (EDEPR), photovoltaic EPR (PVEPR) and photoconductive (PCEPR) detection. EDEPR was found to be more sensitive than EPR by several orders of magnitude. Furthermore, the EDEPR measurements provided more information about the paramagnetic defects at low magnetic fields, such as level crossings of paramagnetic sub-levels, not observable otherwise. The experimental condition for EDEPR observations were investigated such as the dependence on temperature, microwave power and the saturation of the Zeeman levels as well as the influence of shallow dopants on the detection of deep recombination centres.

\section{INTRODUCTION}

Electron paramagnetic resonance (EPR) is a powerful tool for investigating the microscopic and electronic structures of paramagnetic defects in semiconductors and insulators. In semiconductors excess carriers often recombine through recombination centres. In the case of paramagnetic recombination centres, the recombination rate is modified when the EPR transitions of such centres are induced, resulting in a change of the electrical conductivity of the sample, which is believed to occur because of a spin-dependent recombination (SDR) of the charge carriers. Previous investigations showed that the electrical detection of magnetic resonance (EDMR) can be more sensitive than EPR by orders of magnitude. This makes it a very interesting tool to study defects which are present only in low concentrations and therefore not detectable with conventional EPR.

Up to now only a few EDMR investigations have been made on defects in semiconductors, for example on amorphous $\mathrm{Si}$ [1-3], pure $\mathrm{Si}$ [4-10], plastically deformed $\mathrm{Si}$ [11-14], $\mathrm{Si} / \mathrm{SiO}_{2}$ interfaces [15-17] and Si diodes [18-23].

Different methods are known for realising an EDMR experiment. One method is the photoconductive EPR (PCEPR) [8]. During illumination with light the change in photoconductivity of the sample caused by an EPR transition is measured as a change in the resonator quality factor Therefore it is possible to detect EDMR without the use of electrical wires on the sample in a conventional EPR spectrometer.

The direct method to detect an EPR signal is the electrically detected EPR (EDEPR). When inducing the EPR transitions, the change of the conductivity of the sample is measured as a voltage change while keeping the induced dc current through the sample constant [5]. There is also the possibility of photovoltaic detection of EPR signals (PVEPR) for measureing the EDMR of diodes. The illumination of the diode crystal generates a photovoltage, which produces a photocurrent through a load. When driving the EPR transitions, the recombination rate of the excess carriers is enhanced, resulting in a decrease of the photovoltage [25].

Although several models for the spin-dependent recombination process were proposed $[5,24,25]$ the understanding and the knowledge about the mechanism is quite poor. Especially the role of participating defects and defect states, the influence of the Fermi level and shallow donors or acceptors and the defect energy in the band gap are basically not understood.

It is the purpose of this paper to report on different ways to measure EDEPR realised recently in our laboratory and to discuss the present understanding of the mechanisms to detect EDEPR. 


\section{EDEPR INVESTIGATIONS ON DEFECTS IN SILICON DIODES}

First EDEPR results on $\mathrm{Si}$ diodes were published by Solomon et al [21] on IN4005 Si diodes at room temperature. The EDEPR spectrum observed by Solomon was a simple isotropic line similar to that previously observed by Lépine [6] in pure $n$ type bulk $\mathrm{Si}$. From this similarity Solomon conFrom that the same defect causes both spectra for different [6] concluded thes of the sample thickness Lépine due to surface the EDEPR lines observed are dangling bonds. et al [19] on iN4005 EDEPR experiments by Rong slightly different g-values and line $\mathrm{Si}$ diodes show on the manufacturer lines were due to the diode. However, since tons in the bulk of structure was resolved, the no hyperfine (hf) not easy to explain. All those of these lines is carried out at room temperature investigations were EDEPR line without any stucture and show a single

A typical EDEPR structure.

(IN4007, Fagor) is shown in figure 1 a $\mathrm{Si}$ diode

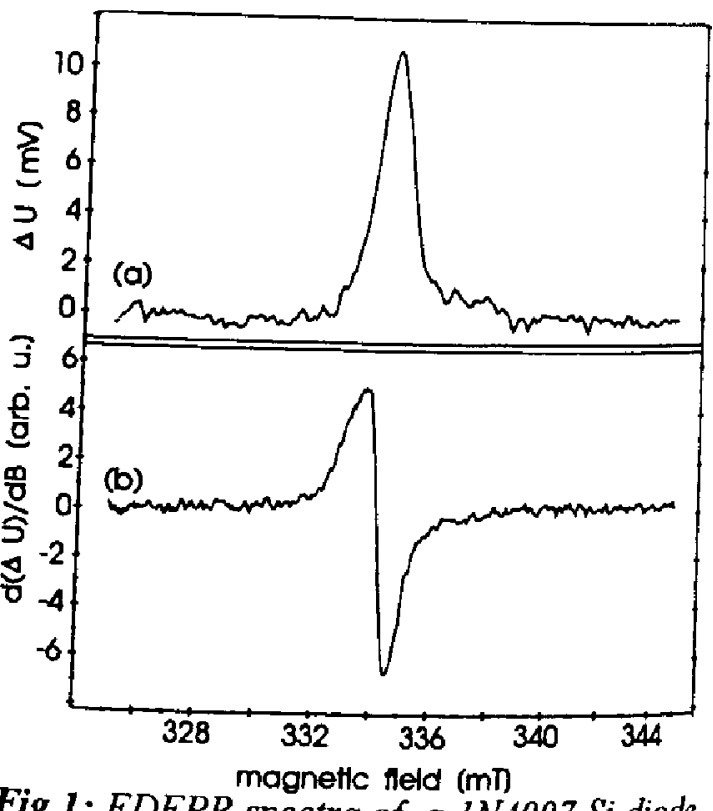

Fig. 1: EDEPR spectra of a $1 N 4007$ Si diode measured at $T=300 \mathrm{~K}$ in the $X$-band (9.36 GHz) with square wave microwave (a) and field modulation (b).

room temperature with a square wave microwave modulation (figure l(a)) and with modulation magnetic field (figure 1(b)) [23]. The EDEPR was observed using a microwave power of $200 \mathrm{~mW}$ at forward injection additional illumination and a nal was a relative current of $1 \mu \mathrm{A}$. The EDEPR sigthe order of $10^{-6}$. Figures 1 (a) and (b) show of there is no significant variation in line width and signal-to-noise ratio when either using microwave or magnetic field modulation. The EDEPR line has the isotropic $g$-value of $2.006(2)$ and a line width of $\Delta \mathrm{B}_{I / 2}=(1.4 \pm 0.2) \mathrm{mT}$. The structural information So far the single line EDEPR spectra is very poor. So far the first angular dependent hf structure was
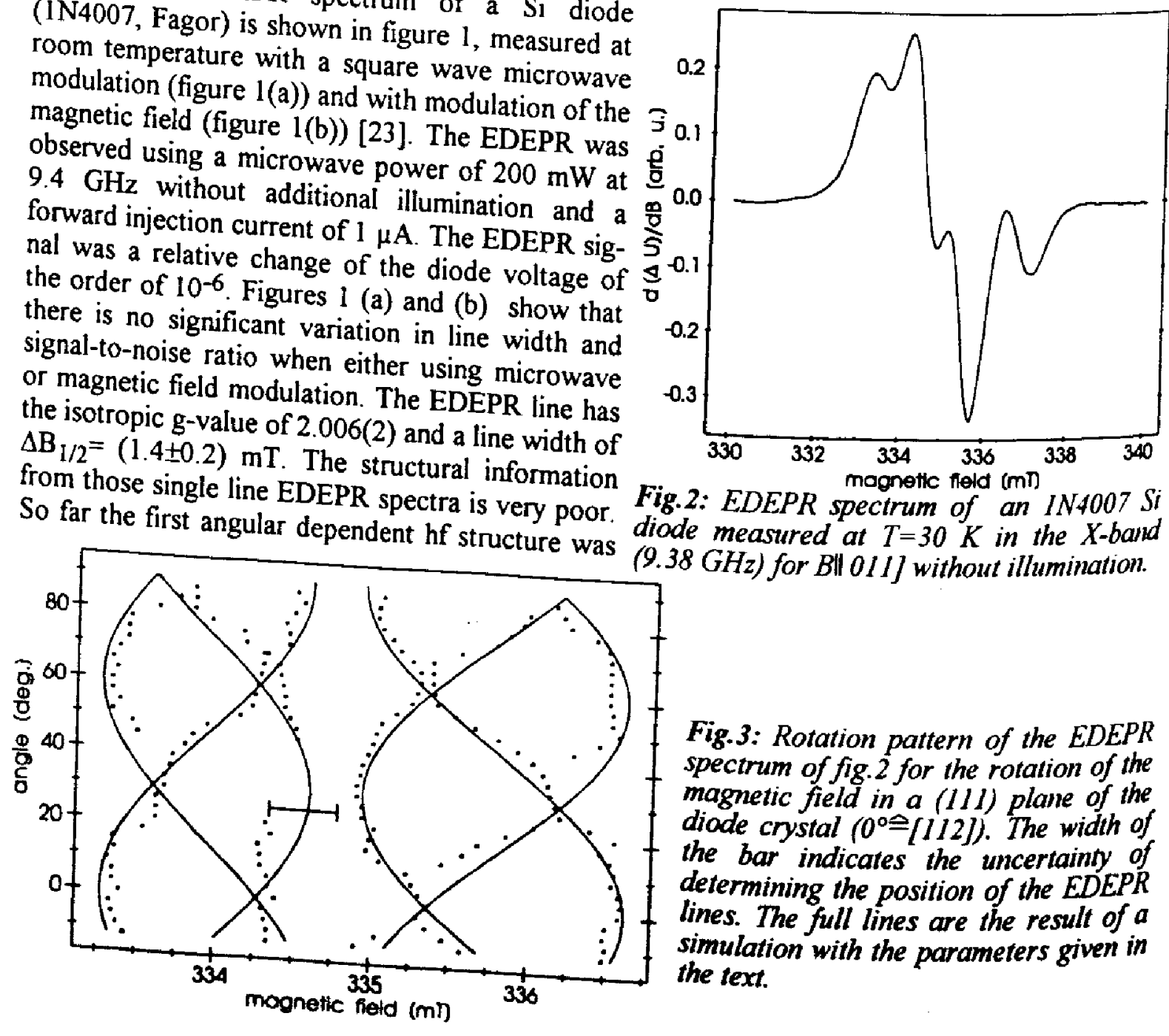

Fig. 2: EDEPR spectrum of an IN4007 Si diode measured at $T=30 \mathrm{~K}$ in the $X$-band (9.38 GHz) for Bll 011] without illumination.

Fig.3: Rotation pattern of the EDEPR spectrum of fig. 2 for the rotation of the magnetic field in a (111) plane of the diade crystal $\left(0^{\circ}=[112]\right)$. The width of the bar indicates the uncertainty of determining the position of the EDEPR lines. The full lines are the result of $a$ simulation with the parameters given in the text. 
observed in a $\mathrm{Si}$ diode at low temperature, caused by a deep recombination centre (figure 2 ). This EDEPR spectrum was observed between $100 \mathrm{~K}$ and $4.5 \mathrm{~K}$ in 1N4007 diodes (Fagor) [23] and is a superposition of at least six lines with a line width $\Delta \mathrm{B}_{1 / 2}$ for a single line of about $0.7 \mathrm{mT}$. The total EDEPR spectrum has a line width $\Delta \mathrm{B}_{1 / 2}$ of about 3 $\mathrm{mT}$ and is centered at $\mathrm{g} 2$. The spectrum of figure 2 was measured after cooling the sample in the dark. Figure 3 shows the rotation pattern of the EDEPR
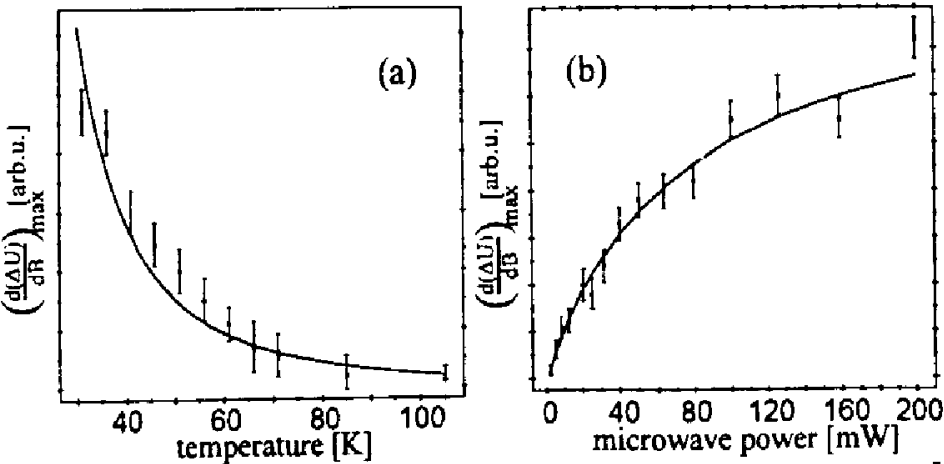

Fig.4: Temperature dependence (a) and microwave power dependence (b) of the EDEPR signal of fig. 2. The bars show the uncertainty of determining the experimental signal intensities, the full lines are fits yielding a temperature dependence as $T^{-2.7}$ (a) and to the power dependence with the measured signal proportional to $\alpha \mathrm{P}_{\mathrm{mw}} /\left(1+\alpha \mathrm{P}_{\mathrm{mw}}\right)$. $\alpha$ is a fit parameter. spectrum for a rotation of the magnetic field in a (111)-plane of the Si diode crystal. The spectrum with at most tetragonal symmetry was analysed with an anisotropic $\mathrm{g}$ - factor $\left(\mathrm{g}_{\|}=2.002, \mathrm{~g}_{\perp}=2.000\right)$ and an anisotropic $h$ interaction $\left(A_{\|}=110 \mathrm{MHz}, A_{\perp}=-20 \mathrm{MHz}\right.$ ) with a nucleus with $I=1 / 2,100 \%$ abundant. On the basis of theoretical calculations of the hf interaction, performed in the general framework of the local spin density approximation to the spin density functional theory, employing a Green's function technique, the EDEPR spectrum was interpreted as being due to a $\mathrm{P}_{\mathrm{i}}-\mathrm{C}_{\mathrm{Si}}$ pair defect [23]. DLTS measurements on diodes which show the low temperature defect yielded three deep energy levels at $E_{C B}-0.25 \mathrm{eV}, \mathrm{E}_{\mathrm{CB}}-0.27 \mathrm{eV}$ and $\mathrm{E}_{\mathrm{CB}}-0.53 \mathrm{eV}$. Diodes of the same type but from different manufacturers, showing no low temperature EDEPR, did not show these DLTS levels. It was assumed therefore that the EDEPR is caused by a deep recombination centre having one of these levels. Neither the EDEPR spectrum at room temperature nor that at low temperatures could be detected with conventional EPR.

The temperature and microwave power dependence of the low temperature EDEPR spectrum show one basic feature of EDEPR, namely that the size of the spin polarisation of the Zeeman levels is not decisive. Figure 4 (a) shows the temperature dependence of one line of the low temperature EDEPR spectrum. The shape of the spectrum does not change with temperature. The variation of the microwave power shows that even at the maximum microwave power level $(200$ $\mathrm{mW}$ ) saturation of the EDEPR signal was not yet achieved (see figure 4 (b)). A good signal could be observed with as low a microwave power as $1 \mathrm{~mW}$. Had a partial saturation with this low power been achieved, then the effect would have been completely saturated at $200 \mathrm{~mW}$. This was not observed: around $200 \mathrm{~mW}$ the EDEPR effect could still be increased significantly. Furthermore, the temperature dependence does not reflect the Boltzmann variation of the spin polarisation of the Zeeman levels for a given microwave transition rate. Saturation of the EPR seems not important. It was found previously that the spin-dependent recombination does not depend on the magnetic field $[11,24]$ and thus not on the Boltzmann equilibrium population of the Zeeman levels.

Another way to detect EDEPR without an injection current is the PVEPR. When illuminating the diode with above-band-gap light, excess carriers are generated and separated in the pn-junction of the diode. The photovoltage then generated can be de-

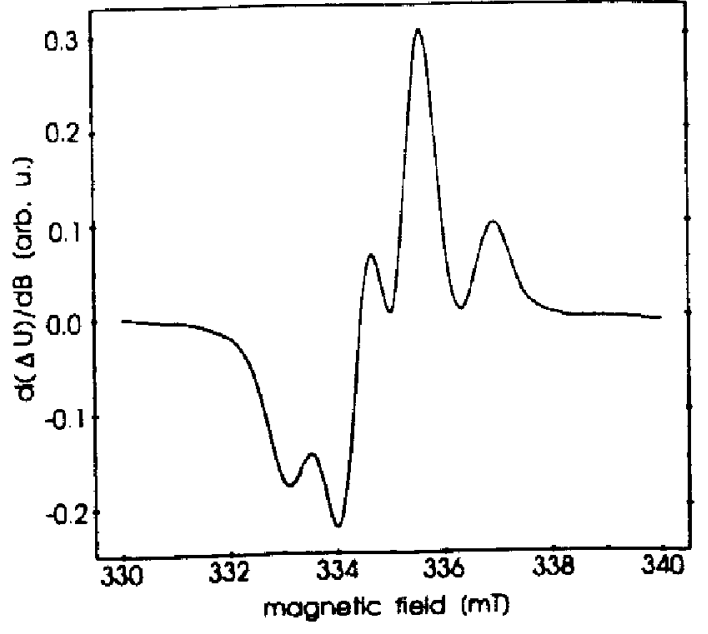

Fig.5: Microwave-induced photovoltaic effect measured in a IN4007 Si diode at $T=30$ $K$ in the $X$-band $(9.38 \mathrm{GHz})$ with illumination with white light of a halogen lamp. 
tected as an EPR signal. Both, the room temperature spectrum (figure 1) and the low temperature spectrum (figure 2) can be measured by PVEPR, showing exactly the same spectra as those measured by EDEPR, except for the phase of the signal, which is inverted. As an example the PVEPR spectrum of the low temperature defect is shown in figure 5 . The fact that the phase of the PVEPR spectrum is inverted in comparison to the EDEPR spectrum is explained as follows. The light creates electrons and holes in the conduction and valence bands, respectively, which move down the diffusion barrier and cause a photovoltaic effect. Losses of electrons and holes occur due to recombination effects, which are enhanced by the EPR transitions, resulting therefore in a decrease of
the photovoltaic effect.

In practice PVEPR is the better choice to measure EDEPR of semiconductor devices, because no constant current or voltage source is needed, which might be an additional source for noise. Moreover, the success of detecting EDEPR critically depends on current-voltage conditions, which can be avoided by measuring PVEPR. In the case of the EDEPR spectra of figures 1 and 2 we observed a sharp maximum at $I=1 \mu \mathrm{A}$ not depending on the temperature $(4-300 \mathrm{~K})$. This observation is in contrast to Christmann et al. [18] and Solomon [21] who concluded from their measurements at $300 \mathrm{~K}$ that the EDEPR depends on the temperature dependence of the current-voltage characteristic of the diode explaining the vanishing EDEPR signal below $300 \mathrm{~K}$. However, the dependence upon the diode current is not connected with the spin-dependent recombination process, since EDEPR can be measured with PCEPR without injection current (see below).

\section{EDEPR INVESTIGATIONS ON DEFECTS IN BULK SILICON}

In bulk Si two different ways to measure EDEPR are known. In both cases one needs to generate the change in carriers by illuminating the sample with above-band-gap light. In order to measure the change in photoconductivity of the sample when inducing an EPR transition one either can across the sample when passing a for of the microwave cavity or directly detect the voltage drop sample. The former method has the advantage that no electrical contacts are needed. Both methods reveal more or less the same sensitivity. In order to study the properties of EDEPR we prepared S
samples with known samples with known defects and investigated them with both methods and for comparison also
with conventional EPR.

Figure 6, trace (a) shows the EPR spectrum of one of those samples, a phosphorus doped CZ-Si

Fig.6: The total time for the measurement, the temperature $(T=6 K)$ and $v_{E P R}=9.48 \mathrm{GHz}$ were the same in all three measurements. All spectra were measured under above-band-gap illumination and normalised to the same signal-to-noise ratio.

(a) Conventional EPR spectrum of an electron-irradiated $P$ doped $S i$ crystal for B\|[110] measured with a microwave power of $0.02 \mathrm{~mW}$. Further details see Text. (b) EPR spectrum of the crystal in figure 6 , trace (a). The concentration of the SLI centre $\left(10^{16} \mathrm{~cm}^{-3}\right)$ was reduced by thermal annealing
to $10^{13} \mathrm{~cm}^{-3}$.

(c) EDEPR spectrum of the crystal of figure 6 , trace (b). The orientation of the sample was $B \mid[[110]$ $+3^{\circ}$ and the microwave power was $200 \mathrm{~mW}$.

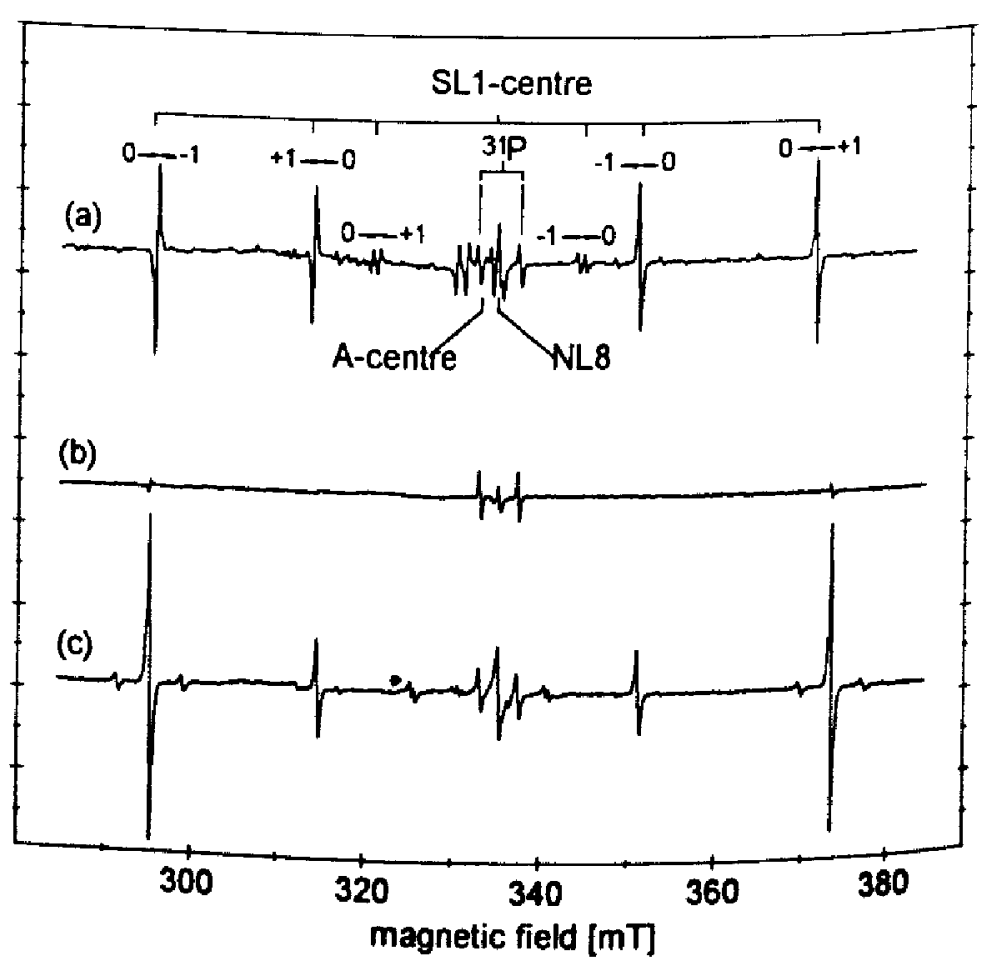


crystal (P: $8.5 \cdot 10^{14} \mathrm{~cm}^{-3}, 3 \times 4 \times 5 \mathrm{~mm}^{3}$ ), which was electron irradiated. The spectrum reveals therefore the resonance lines of the A centre, a negatively charged state of the oxygen-vacancy pair with $\mathrm{S}=1 / 2[27]$ and lines of the SLl centre, an excited triplet state of the oxygen-vacancy pair with $S=1[28]$. The latter one is only observed after illumination with transitions labelled by 'SLI' in figure 6 , trace (a), which are centred around $g \approx 2$ and show transitions between different $m_{s}$ states. The transitions $|-1>\leftrightarrow| 0>$ and $|0>\leftrightarrow|+1>$ are inverted in phase, because of an inversion of level occupation of the SLl triplet [30]. The concentration of the irradiation centres was $1 \cdot 10^{16} \mathrm{~cm}^{-3}$. Phosphorus gives rise to two EPR lines with the characteristic hf splitting of $4.2 \mathrm{mT}$ (figure 6, trace (a)). Besides thermal donors were created with thermal treatment at temperatures of $T \approx 450^{\circ}$ C [29], also observed in the EPR spectrum of figure 6, trace (a), labeiled 'NL.8'. For the EPR measurements the largest microwave power of $200 \mathrm{~mW}$ was attenuated by $40 \mathrm{~dB}$, because then the best signal-to-noise ratio was achieved. We reduced the concentration of the irradiation defects by annealing the sample in order to demonstrate that EDEPR can be more sensitive than EPR. After annealing $30 \mathrm{~min}$. at $350^{\circ} \mathrm{K}$ we observed the spectrum shown in figure 6 , trace (b). Due to annealing the oxygen centres were partely destroyed resulting in the disappearance of the A-centre lines. The resonance lines of the SLl centre are very weak, while those of the NL8 and P remained unchanged. Besides detecting EDEPR via the change in quality factor of the microwave resonator we used the direct method to measure the change in photoconductivity, by measuring the voltage drop across the sample when passing a constant current through it. To this end electrical contacts were added onto one side of the sample. EDEPR measurements were carried out with $200 \mathrm{~mW}$ microwave power. For EDEPR we generated a dc current of $5 \mu \mathrm{A}$ through the sample, which produced a voltage drop of about $0.5 \mathrm{~V}$ across the sample. In the dark no EDEPR spectrum was observed. Under band-gap illumination we observed the EDEPR lines of P, NL8 and SL1 (figure 6, trace (c)). In the EDEPR spectrum the line intensity ratios of the $P$ lines and thermal donors are different compared to those observed by EPR. The P signals show the same signal-to-noise ratio in EPR and EDEPR, whereas for NL8 this ratio is more than three times larger in EDEPR than in EPR. Moreover, the EDEPR shows intense lines for the SL1 centre, which were hardly detectable by EPR. The signal-to-noise ratio of EDEPR was large enough to observe a hf structure due ${ }^{29} \mathrm{Si}$ of the SL1 centre $\left({ }^{29} \mathrm{Si}, \mathrm{I}=1 / 2,4.7 \%\right.$ abundance). Due to absorption of above-band-gap light EDEPR is measured only in a thin surface layer (see below) in constrast to EPR. Therefore the absolute number of defects detected with EDEPR was less than that detected with EPR by a factor of 600 . We found that the sensitivity for NL8 measured with EDEPR was increased by a factor of 3 in comparison to conventional EPR. In case of the shallow thermal donors EDEPR is therefore approximately $10^{3}$ more sensitive than conventional EPR, which is comparable with the sensitivity of optically detected EPR (ODEPR). However, the sensitivity enhancement is different for different defects.

In order to study the EDEPR effects seen in the spectrum of figure 6 , trace $(c)$, we prepared several samples containing these defects separately. The bulk Si crystals were investigated with EDEPR using electrical contacts and with conventional EPR. One sample containing $\mathbf{P}$ only with a concentration of $8.5 \cdot 10^{14} \mathrm{~cm}^{-3}$ shows the known conventional EPR spectrum of the shallow $P$ donor (see above), but did not show any EDEPR spectrum. A boron doped (4.5. $10^{15} \mathrm{~cm}^{-3}$ ) fz oxygen diffused sample containing $3 \cdot 10^{15} \mathrm{~cm}^{-3}$ thermal donors shows under illumination the EPR spectrum NL8, which also was observed with EDEPR (see figure 7). However, the shallow thermal donors (NL8) seem to act as recombination centres

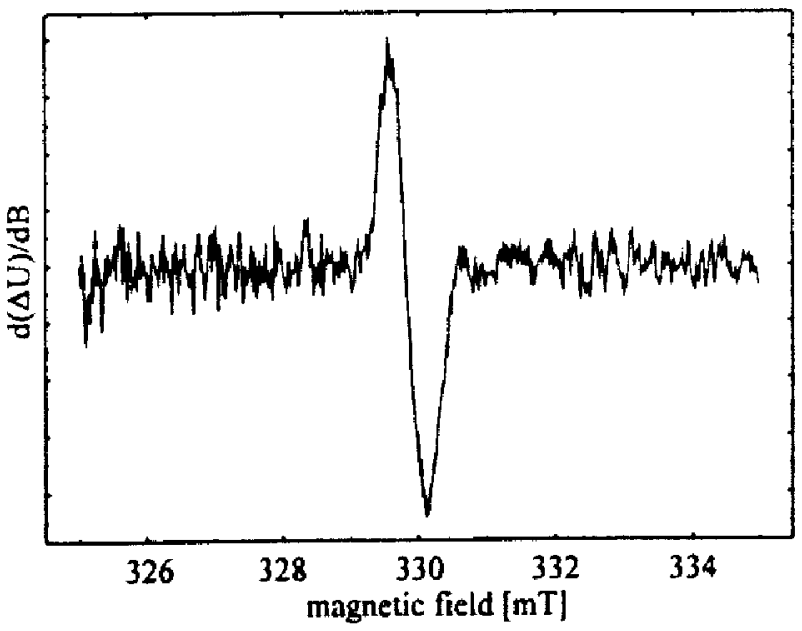

Fig. 7: EDEPR spectrum of a boron doped $f z$ oxygen diffused $\mathrm{Si}$ crystal showing under illumination the resonance of $N L 8 . T=20 \mathrm{~K}$, $B||[100]$. 
or it is the presence of the boron acceptor which enables a spin-dependent recombination process (see below).

One basic property of EDEPR is that the signal intensity is independent of the magnetic polarisation of the paramagnetic defects as observed, for example, by Mima [11] in plastically deformed silicon at $9.3 \mathrm{GHz}$ and at $2.4 \mathrm{GHz}$ and as concluded from the microwave power and temperature dependence of the $\mathrm{P}_{\mathrm{i}}-\mathrm{C}_{\mathrm{s}}$ defect in a $\mathrm{Si}$ diode (see above). In order to test a microwave frequency dependence. for the defects causing the EDEPR of figure 6 , trace (c), we carried out EDEPR measurements at low resonance frequencies $\left(v_{\mathrm{EDEPR}}=400 \mathrm{MHz}\right)$. The low-frequency spectrum shown in figure 8 con-

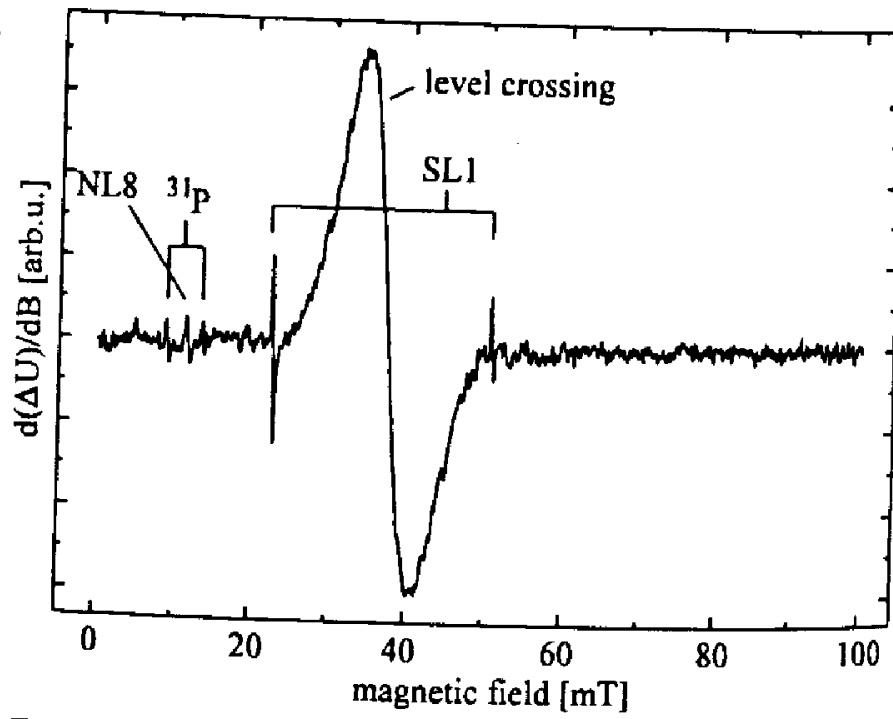

Fig.8: Low field EDEPR spectrum of a phosphorus doped CZ Si crystal containing NL8 and SLI centres under above-band-gap illumination. $v_{E D E P R}=400 \mathrm{MHz}$, sists of one broad line and several nar$T=6 \mathrm{~K}$ and $B||[110]+5^{\circ}$. between magnetic sub-levendent recombination enables one to detect non-resonant transitions non-resonant transition can be spin system as a change in the conductivity of the sample. Such a Zeeman level crossing of the SLI $\mid+1>$ and $\mid 0>$ levDEPR spectrum of the SLI centre due to a wave field. Hence this EDEPR line can be atribuels. The line appears without a resonant micromicrowave induced EPR transitions. By changittributed to a non-resonant transition in contrast to $B \|[011]$ a line broadening and a strong ding the magnetic field orientation of the sample from orientation of $\mathrm{B} \|[011]+10^{\circ}$ only a small broad line is left. Besides the non-resonant transition of the (see figure 8). They were weak, because to P, thermal donors and the SL1 centre were observed We studied the microwave power depend the small power of about $1 \mathrm{~mW}$ available at $400 \mathrm{MHz}$ $\mathrm{mW}$ and $200 \mathrm{~mW}$ and estimated that thence of the SL1, NL8 and P lines at $9.5 \mathrm{GHz}$ between 10 $\mathrm{mW}$ is expected. Obviously the EDEPR eff-to-noise ratio of the lines in figure 8 measured at 1 Zeeman levels.

A still open question is related to the defect concentration needed to observe EDEPR. An the SLl defect given by Vlasenko et al. [30] for EPR could not be measured that EDEPR and However, this was not observed the same time. (see figures 6, traces (b) and (c)) in our study thermal donors with concentrations $P$ and the EPR detection were for therefore conclude observed by EDEPR. We low defect concentrat EDEPR is not limited to centrations.

detectable by believed that EDEPR is so far not known. It is orders of magnitude more be at least about two order to get a quantitative limit, we used the same electron- for a lower sample as above (figure 6, trace (c)) with electri-

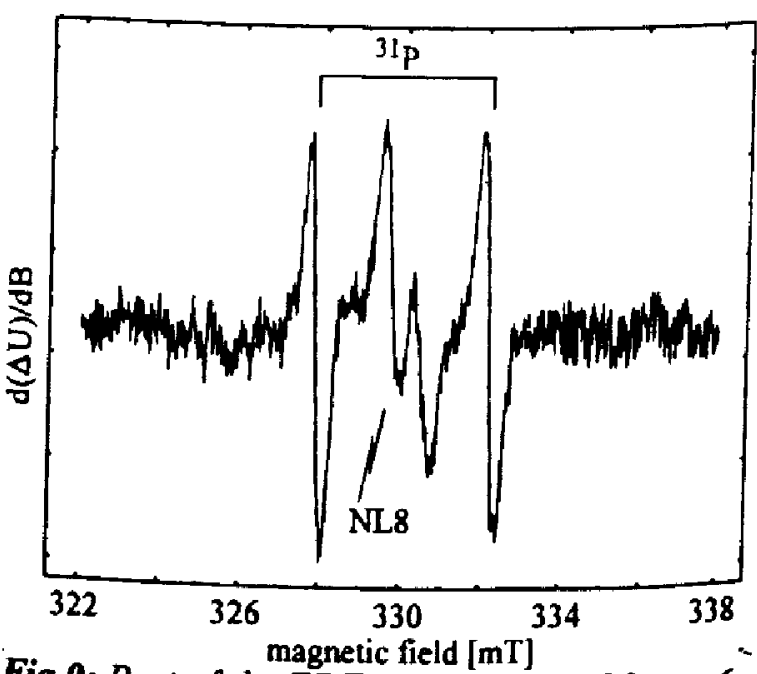

Fig.9: Part of the EDEPR spectrum of figure 6 , trace (c). Only a spot of $0.6 \mathrm{~mm}$ diameter was illuminated with a HeNe LASER. T=20 K, $B \mid[I I I]$ 
cal contacts and illuminated the sample with a HeNe LASER. Because of nearly complete absorption of light with $\mathrm{E}>\mathrm{E}_{\mathrm{gap}}$ in the surface region of the silicon sample, photoconductivity is produced only in a rather small layer of approximately $5 \mu \mathrm{m}$ thickness. First we carried out EDEPR measurements on the electron-irradiated silicon crystal, while illuminating only the sample face fitted with the electrical contacts. Then the measurements were repeated while illuminating the back side of the sample. We were able to detect the EDEPR spectrum only when illuminating the sample face fitted with the electrical contacts. Otherwise no EDEPR spectrum appeared. This shows that EDEPR is only measured in a small surface layer of the sample.

We then illuminated the face of the crystal where the contacts were fitted with a small spot of 0.6 mm diameter with a HeNe LASER $(50 \mathrm{~mW})$. In this way we detect an absolute number of $P$ defects of about $4 \cdot 10^{8}$. (The concentration of $P$ was $8.5 \cdot 10^{14} \mathrm{~cm}^{-3}$.) Figure 9 shows a part of the EDEPR spectrum with $P$ and NL8 centres. The signal-to-noise ratio was 12 and therefore large enough to detect the EDEPR of about $5 \cdot 10^{7}$ defects. The signal-to-noise ratio can be improved by using a LASER providing more power. The fact that EDEPR can be observed within a small part of the sample surface illuminated, enables one to investigate the distribution of recombination centres in a sample surface layer with high sensitivity.

\section{DISCUSSION OF SPIN-DEPENDENT RECOMBINATION}

The observation of EDEPR is usually explained with a spin-dependent recombination of excess carriers at the recombination centre. Several models have been proposed. For example, Lépine et al. $[5,6]$ suggested that there is a spin-dependent electron capture cross section at the recombination centre, where electrons and holes are supposed to recombine, an effect which was thought to depend on the spin polarisation of the paramagnetic centre. In order to decrease the conductivity of the sample the EPR of the recombination centre must be saturated, i.e a deviation of the Boltzman occupation of the Zeeman levels must be achieved by the microwave transitions. However, we showed that the EDEPR effect does not depend on the spin-polarisation, in line with Kaplan et al [24] and Mima et al. [11].

In the spin-dependent Shockley Read model proposed recently by Rong et al. [25] the electron is captured in an excited state of the recombination centre before it relaxes spin-dependently into the ground state and recombines with a hole. According to this model the EPR of the excited states should have been observed too. We did not observe such an excited state. We have only observed the ground state of the paramagnetic defect. On the other hand the spin-dependent recombination must occur at the recombination centres, since we see their characteristic properties. It must even be 'sensitive' to nuclear Zeeman levels since we see the hf structure.

Lanoo et al [31] proposed a model in which the spin-dependent recombination is explained with a bound exciton associated to a neutral defect. Furthermore spin-dependent and conventional nonradiative recombination were considered. However, in one of our crystals the Fermi level was above $P$ and therefore neutral $P$ was observed with conventional EPR without illumination, but the signals were not detectable with EDEPR. For neutral $P$ the bound exciton $\left(\mathrm{P}^{0} \mathrm{X}\right)$ is known, having a binding energy of $5 \mathrm{meV}$ [32]. According to the model of Lanoo et al. we should have observed EDEPR in such a crystal. This was not the case.

With the models published so far to explain the spin-dependent recombination effect observed in EDEPR we cannot explain our observations satisfactorily.

So far our EDEPR observation in bulk Si may be described within a donor-acceptor recombination model. NL8 $\left(3 \cdot 10^{15} \mathrm{~cm}^{-3}\right)$ in boron doped Si $\left(4.5 \cdot 10^{15} \mathrm{~cm}^{-3}\right)$ was observed with EPR only under illumination, since the Fermi level was below NL8. Therefore NL8 was observed with EDEPR due to creation of electrons and holes by above-band-gap light assuming that the unoccupied NL8 captures an electron, which recombines with the hole captured by the boron acceptor. EPR of acceptors in $\mathrm{Si}$ is usually not observed [33]. It is in line with this model that in a sample containing $P$ only, no EDEPR can be observed, sine the P level is occupied. The Si crystal containing P, NL8 and SLl (see figure 6) shows EPR only under illumination. However, the observation of EDEPR assumes the presence of an acceptor, which might be present due to electron irradiation. A more 
complicated or different recombination path in this case cannot be excluded. In order to discover the EDEPR effects it seems more sensible to investigate known defects than unknown defects in
devices as done before

1) I. Solomon, D. Biegelsen and J.C. Knights, Solid State Communications, 1977, 22 pp505-508

2) R.A. Street, Philosophical Magazine B , 1982, 4646 pp273-278

Phys., 1983, 16 L427-L431

4)

5) D.J.ép, Phys. Rev. Lett., 1966, 17 (4) pp186-188

Physics of Semiconductors in Proceedings of the ninth International Conference on the

6) D.J. Lépine, Phys. Rev. B, Boston, 1970, pp805-807

7) G. Mendz and D.J. Haneman, 1972,62 pp436-441

8) G. Mendz, D.J. Miller and D, Phys. C: Solid State Phys., 1978, 11 L197

9) V.V. Kurylev and S.N. Karyagin phys, Phys. Rev. B, 1979, 2012 pp5246-5251

10) V.S. L'vov, O.V. Tretyak and A phys. stat. sol. (a), 1974, 21 K127

$11)$ L.S. Mima, V.I. Strikha and O.A. Kolomiets, Sov. Phys. Semicond., 1977, 11 (6) pp661-664

12) V.S. L'vov, L.S. Mima and O.V. Tretyak, Sov. Phys. Semicond, 1980, 14 pp1328-1329

13) V.A. Grazhulis, V.V. Kveder and Tretyak, Sov. Phys. JETP, 1982, 56 (4) pp897-902

14) D. Neubert, K. Hoffman, H Teichmu A. Osip'yan, JETP Lett., 1975, 21 (12) 335

15) P.M. Lenahan and M.A. Jupinat Teichmann and R. Schlief, Solid State Electronics, 1978, 211445

16) B. Henderson, M. Pepper and, Colloids and Surfaces, 1990, 45 pp 191-211

17) V.R. Vranch, B. Henderson M.L. Vranch, Semicond. Sci. Technol., 1989, 41045

18) P. Christmann, C. Wetzel, B K. Pepper, Appl. Phys. Lett., 1988, 52 ppl161-1163 (15) pp1857-1859

19) F.C. Rong E.H Poin Commumications

20) F.C. Rong, G.J. Ger 1990, 768 pp 1083-1086

W.L. Warien, Appl. Phy. W. Buchwald, E.H. Poindexter, M.T. Umior, D.J. Keeble and

21) I. Solomon, Solid State Comm. Lett. 1992, 60 (5), pp610-612

22) F.I. Borisov, V.I. Strikha and Onications, 1976, 20 pp215-217

23) B. Stich, S. Greulich-Weber, H. Tretyak, Sov. Phys. Semicond., 1981, 15 (10) ppl 149-1151 in press

24) D. Kaplan, I. Solomon and N.F. Mott, Le Journal de Physique Lettres, 1978, 39 (4) L51-L54 25) F.C. Rong, W.R. Buchwald, E.H. Poindexter, W.L. Warren and D.J. Keeble, Solid State
Electronics, 1991, 348 pp835-841

27) G.D. Cavenett and R.F. Brunwin, Solid State Communications, 1979, 31 pp659-662

28) K.L. Brower, Phys. Rev. $B$. 1971, Phys. Rev., 1960, 1214 pl001-1014

29) J. Michel, J.R Niklas and 1971, 46 pp 1968-1982

30) L.S. Vlasenko, M.P. Vlasenko, VN. Shys. Rev. B, 1988, 38, 3998 1986, (3) pp612-619

31) M. Lannoo, D. Vuillaume, D. Deresmes and D. Stiévenard, 1993, private communication

32) M.A. Vouk and E.C. Lightowlers, 1977, J. Lumin. 15, 17

33) G.W. Ludwig and H.H. Woodbury, 1962, Solid State Physics 13 pp223, ed. F. Seits and
D. Turnbull 